\title{
Moderately hypofractionated carbon ion radiotherapy for prostate cancer; a prospective observational study "GUNMA0702"
}

\author{
Hidemasa Kawamura ${ }^{1,2^{*}}$ (D, Nobuteru Kubo ${ }^{1,2}$, Hiro Sato ${ }^{1}$, Tatsuji Mizukami ${ }^{1}$, Hiroyuki Katoh ${ }^{3}$, Hitoshi Ishikawa $^{4}$, \\ Tatsuya Ohno ${ }^{1,2}$, Hiroshi Matsui 1,5, Kazuto Ito ${ }^{5,6}$, Kazuhiro Suzuki ${ }^{1,5}$, Takashi Nakano ${ }^{1,2}$ and Group for Genitourinary \\ Tumors at Gunma University Heavy Ion Medical Center
}

\begin{abstract}
Background: Carbon ion Radiotherapy for prostate cancer is widely used, however reports are limited from single institute or short follow up. We performed a prospective observational study (GUNMA0702) to evaluate the feasibility and efficacy of carbon ion radiotherapy for localized and locally advanced prostate cancer.

Methods: Between June 2010 and August 2013, 304 patients with localized prostate cancer were treated, with a median follow-up duration of 60 months. All patients received carbon ion radiotherapy with $57.6 \mathrm{~Gy}$ (RBE) in 16 fractions over 4 weeks. Hormonal therapy was given according to the risk group. Toxicity was reported according to the Common Toxicity Criteria for Adverse Event, Version 4.0 by the National Cancer Institute.
\end{abstract}

Results: The overall 5-year biochemical relapse-free rate was 92.7\%, with rates of 91.7, 93.4, and 92.0\% in low-risk, intermediate-risk, and high-risk patients, respectively. The 5-year local control and overall survival rates were 98.4 and $96.6 \%$, respectively. Acute grade 3 or greater toxicity was not observed. Late grade 2 and grade 3 genitourinary and gastrointestinal toxicity rates were 9 and $0.3 \%$, and 0.3 , and $0 \%$, respectively.

Conclusions: The present protocol of carbon ion radiotherapy for prostate cancer provided low genitourinary and gastrointestinal toxicity with good biochemical control within 5 years.

Trial registration: University Medical Information Network Clinical Trial Registry number: UMIN000003827.

Keywords: Prostate cancer, Carbon ion radiotherapy, Prospective study

\section{Background}

The incidence of prostate cancer has increased in some European countries [1] and is increasing in Japan, Singapore, and China [2]. The number of patients diagnosed with localized prostate cancer is also increasing because of prostatespecific antigen (PSA) screening. Localized prostate cancer must be managed to maintain quality of life because they are not a life-threatening cancer in many cases. Radiation

\footnotetext{
*Correspondence: kawa@gunma-u.ac.jp

${ }^{1}$ Gunma University Heavy lon Medical Center, 3-39-22, Showa-machi,

Maebashi, Gunma 371-8511, Japan

${ }^{2}$ Department of Radiation Oncology, Gunma University Graduate School of

Medicine, Maebashi, Gunma, Japan

Full list of author information is available at the end of the article
}

therapy could be an excellent treatment option for localized prostate cancer due to its effectiveness and low incidence of adverse events. Recently, the use of radiation for treating prostate cancer has increased by approximately $10 \%$ compared with previous Japanese studies [3].

Carbon ion radiotherapy (CIRT) has been used for localized and locally advanced prostate cancer. CIRT may represent an ideal treatment method for prostate cancer due to the unique physical and biologic advantages of carbon ion beams. The dose distribution of CIRT for prostate cancer is most advantageous for external beam irradiation techniques because of its superior dose characteristics [4]. Moreover, carbon ion beams have a high relative biological effectiveness (RBE), resulting from a high linear energy transfer, 
with their effect estimated to be approximately three times those of photons and protons $[5,6]$. The first clinical trial of CIRT for prostate cancer was initiated at National Institute of Radiological Sciences (NIRS) in 1994. The efficacy and feasibility of CIRT for localized prostate cancer have been demonstrated through three phase I/II and two phase II clinical trials [7]. Appropriate dose fractionation schedules for CIRT and use of androgen deprivation therapy (ADT) according to tumor risk groups have also been reported. However, all previous reports of the use of this treatment are from the single institution; therefore, further trials by other institutions are required to validate these outcomes.

In 2009, Gunma University Heavy Ion Medical Center (GHMC) installed a new compact-sized accelerator and began providing CIRT since March 2010 [8]. Herein, we performed a prospective observational study (GUNMA 0702) to confirm the efficacy and toxicity of CIRT for localized prostate cancer.

\section{Methods}

Patient eligibility

Patients aged between 20 and 80 years with histologically confirmed adenocarcinoma of the prostate staged as T1-T3N0M0 according to the International Union Against Cancer TNM classification (2002) were eligible for the present study. Assessments of Gleason Score (GS) were centrally reconfirmed for all tumors prior to study registration. Tumor stage was determined based on digital examination, trans-rectal ultrasound, computed tomography $(\mathrm{CT})$, magnetic resonance imaging (MRI), and bone scanning. Exclusion criteria were history of pelvic RT or the presence of concurrent active malignancies or inflammatory disease. Patients who had received previous treatments for prostate cancer were also excluded.

\section{Central pathology review}

All biopsy specimens were centrally re-evaluated by one pathologist at Gunma University Hospital. Tumor grade was assigned according to the modified Gleason grading system proposed by the International Society of Urological Pathology.

\section{Risk classification}

Patients were stratified into three subgroups according to three major clinical risk factors of prostate cancer: T stage; initial prostate-specific antigen (iPSA) value; and GS. If patients with T1c-T2bN0M0 diseases had an iPSA $<10$ $\mathrm{ng} / \mathrm{ml}$ and $\mathrm{GS} \leq 6$, they were allocated to the low-risk group. In contrast, patients with T3 or iPSA $\geq 20 \mathrm{ng} / \mathrm{ml}$ or $\mathrm{GS} \geq 8$ were assigned to the high-risk group. The remaining patients were classified as the intermediaterisk group.

\section{Androgen deprivation therapy}

ADT consisted of medical castration by LH-RH agonists with or without anti-androgen and was administered according to our risk group criteria. For patients in the low-risk group, CIRT was performed without the use of ADT. Neo-adjuvant ADT was administered to patients in the intermediate- and high-risk groups for 5-8 months before the initiation of CIRT. Adjuvant ADT without anti-androgen was continued for high-risk patients only, and ADT was administered for a total of 24 months. Patients with $\mathrm{T} 1 \mathrm{c}-\mathrm{T} 2 \mathrm{~b}$ prostate cancer who had a GS of $7(3+4)$ and an iPSA value less than $10 \mathrm{ng} /$ $\mathrm{ml}$ were exceptionally regarded as having intermediaterisk but received CIRT without ADT.

\section{Carbon ion radiotherapy (CIRT)}

A similar technique of CIRT for prostate cancer, which has been reported from NIRS, was used in the present study [7]. The feet of patients were positioned in a customized cradle (Moldcare; Alocare, Tokyo, Japan) and the pelvis was immobilized with a low-temperature thermoplastic sheet (Shellfitter; Kuraray, Co., Ltd., Osaka, Japan). At CT simulation, the bladder was filled with $100 \mathrm{~mL}$ sterilized saline and the rectum was emptied using an enema.

Treatment planning was performed using CT images of $2 \mathrm{~mm}$ thickness with fused MRI images with Xio-N (Elekta, Stockholm, Sweden and Mitsubishi Electric, Tokyo, Japan) [8]. The clinical target volume (CTV) included the prostate and the proximal seminal vesicles (SV). In T3b cases, we include the part of seminal vesicle as CTV where was involved by prostate cancer at diagnosis (pre neoadjuvant hormonal therapy) at least. The initial planning target volume (PTV1) was created by adding the anterior and lateral margins of $10 \mathrm{~mm}$, cranial and caudal margins of $6 \mathrm{~mm}$, and a posterior margin of $5 \mathrm{~mm}$ to the CTV, with lateral margins to the $\mathrm{SV}$ of $3 \mathrm{~mm}$. According to the protocol from the NIRS, boost therapy was performed using the second PTV (PTV2), in which the posterior edge was set in front of the anterior wall of the rectum after the completion of nine fractions while the other margins remained the same as for PTV1 [9]. Each field was defined using spread-out Bragg peak and shaped by multi-leaf collimators and compensation bolus for each patient.

CIRT was performed at a total dose of $57.6 \mathrm{~Gy}$ (RBE) in 16 fractions over 4 weeks, with a fractional dose of 3.6 Gy (RBE) at four fractions a week. One field was used for each session, including one anterior field and a pair of lateral ports for PTV1 and another pair of lateral ports for PTV2. The bladder was also filled with $100 \mathrm{~mL}$ sterilized saline at each treatment session from the anterior direction. Patient positioning was three-dimensionally corrected using the same treatment couch used at the NIRS. All treatment plans were approved by the institutional conference prior to administering treatment. 


\section{Assessment}

Follow-up evaluations, including physical examination, blood testing for serum PSA, and urine screening, were performed at 3-month intervals. CT and MRI were performed once a year. Acute and late toxicities were evaluated using Common Terminology Criteria for Adverse Events version 4.0 (National Cancer Institute).

\section{Statistical considerations}

The primary objective of the present study was bRFR at 5 years. Previous reports of bRFR at 5 years with X-ray IMRT were $91 \%$ in low-risk patients, $76 \%$ in intermediate-risk patients, and $68 \%$ in high-risk patients [10]. The reported bRFR with CIRT at the NIRS were $89 \%$ in low-risk patients, $97 \%$ in intermediate-risk patients, and $84 \%$ in high-risk patients [11-13]. Based on the assumption that the proportions of patients in lowrisk, intermediate-risk, and high-risk groups are 6, 47, and $47 \%$, respectively, 266 patients were planned to be enrolled at an overall type I error rate of $\alpha=0.05$ and power of $1-\beta=0.80$.

Actuarial analysis was used to determine biochemical relapse-free survival by the Kaplan-Meier method. The OS, CSS, LCR, and bRFR were calculated from the CIRT start date. Biochemical failure was defined according to Phoenix criteria, as a rise of $>2.0 \mathrm{ng} / \mathrm{mL}$ above the PSA nadir [14].

\section{Results}

\section{Patients accrual and registration}

The present study was conducted from June 2010 to August 2013; a total of 309 patients were enrolled. The average monthly accrual for the study was 12.4 patients.

Two patients were judged as ineligible due to registration process errors, i.e., they were aged $>80$ years at the time of registration. Three patients were registered but did not receive CIRT: two patients withdrew on their own after registration, and one patient developed renal infarction prior to the start of CIRT (Fig. 1). During the analysis of the 304 eligible patients, follow-up information was available for all patients. All patients in the present analysis had completed the scheduled CIRT. Three patients stopped ADT before the protocol regulated period due to unbearable toxicity and two patients received prolonged ADT with consent between patients and urologists.

\section{Patient characteristics}

Table 1 lists the pretreatment characteristics of the 304 eligible patients. The median age of the patients was 66 years (range, 48-80 years). The majority of patients were stratified into the intermediate- $(47 \%)$ or high-risk (48\%) group, with only $5 \%$ of patients stratified into the low-risk group. Of these patients, $78 \%$ received ADT. The median follow-up period for surviving patients was 60.4 months.

\section{Treatment results}

The overall 5-year bRFR was 92.7\% (95\% CI: 89.7-95.7\%; Fig. 2a), and 91.7, 93.4, and $92.0 \%$ in low-risk, intermediate-risk, and high-risk patients, respectively (Fig. 2b). bRFR for intermediate-risk patients treated with CIRT alone and CIRT plus hormonal therapy were 97.5 and 91.1\%, respectively (Fig. 2c). Local recurrence was observed in $4(1.3 \%)$ patients, with a 5 -year LCR of $98.4 \%$ (95\% CI: 96.8-100\%; Fig. 3). Distant metastasis was observed in $6(2.0 \%)$ patients. Three patients $(1.0 \%)$ died of prostate cancer and 9 (3.0\%) died from other causes, with an OS rate of $96.6 \%$ (95\% CI: 94.5-98.7\%; Fig. 4).

\section{Toxicity}

Table 2 shows acute and late toxicities following CIRT. Analysis of acute toxicities showed that the incidence of G2 and G3 genitourinary (GU) toxicities was 4.0, and

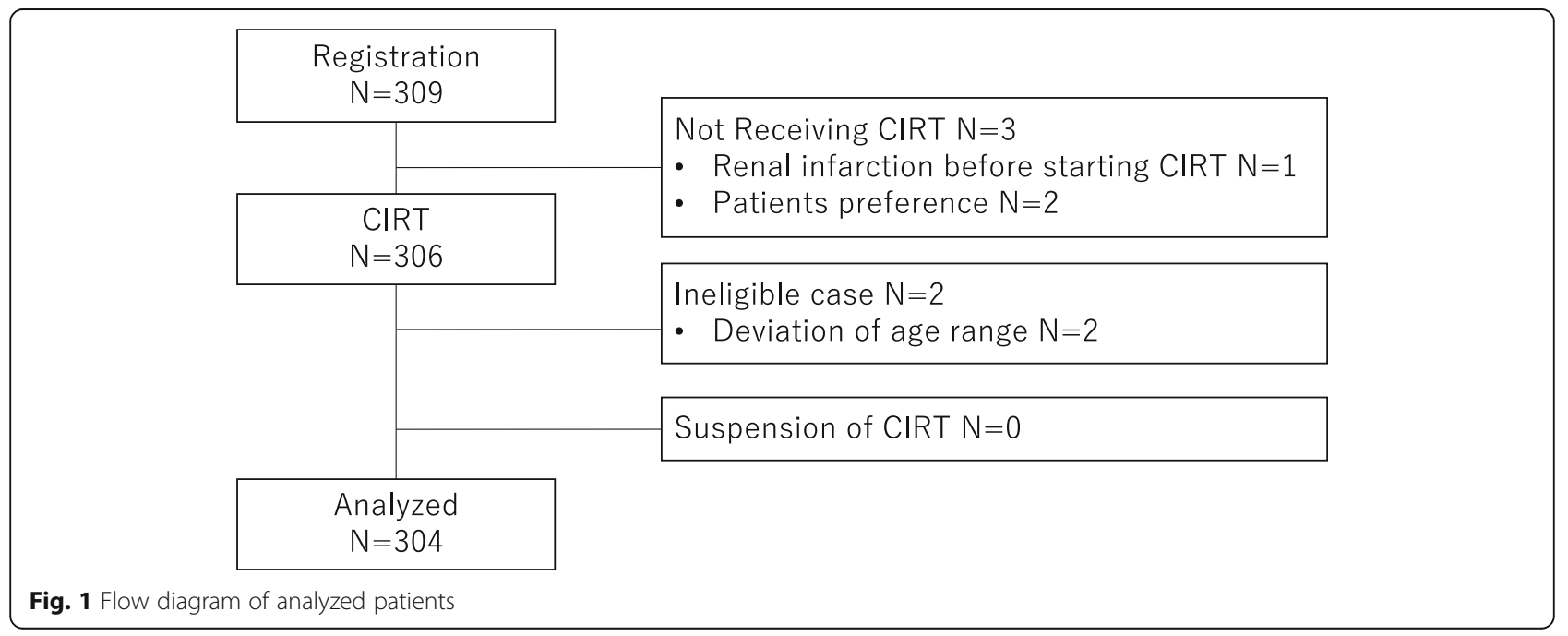


Table 1 Patient Characteristics

\begin{tabular}{|c|c|}
\hline Age (year) & $66(48-80)$ \\
\hline \multicolumn{2}{|c|}{ T stage (UICC 2009) } \\
\hline T1c & $83(27 \%)$ \\
\hline$T 2 a-b$ & $86(28 \%)$ \\
\hline $\mathrm{T} 2 \mathrm{C}$ & $60(20 \%)$ \\
\hline Т3а & $69(23 \%)$ \\
\hline $\mathrm{T} 3 \mathrm{~b}$ & $6(2 \%)$ \\
\hline \multicolumn{2}{|l|}{ Gleason Score } \\
\hline 6 & $20(7 \%)$ \\
\hline 7 & $171(56 \%)$ \\
\hline $3+4$ & 97 (32\%) \\
\hline $4+3$ & 74 (24\%) \\
\hline 8 & $36(12 \%)$ \\
\hline 9 & $71(23 \%)$ \\
\hline 10 & $6(2 \%)$ \\
\hline \multicolumn{2}{|l|}{ PSA (ng/ml) } \\
\hline$<10$ & $195(64 \%)$ \\
\hline $10-20$ & $75(25 \%)$ \\
\hline$\geqq 20$ & $33(11 \%)$ \\
\hline \multicolumn{2}{|l|}{ Risk group } \\
\hline Low & $16(5 \%)$ \\
\hline Intermediate & $142(47 \%)$ \\
\hline High & $146(48 \%)$ \\
\hline \multicolumn{2}{|l|}{ ADT } \\
\hline No & $66(22 \%)$ \\
\hline Yes & $238(78 \%)$ \\
\hline
\end{tabular}

$0 \%$, respectively, and the incidence of G2-G3 gastrointestinal (GI) toxicities was $0 \%$. Analysis of late toxicities revealed that the incidence of $\mathrm{G} 2$ and $\mathrm{G} 3 \mathrm{GU}$ toxicities was 9 and $0.3 \%$, respectively, and the incidence of G2 and G3 GI toxicities was 0.3 and $0 \%$, respectively. One patient experienced bladder bleeding requiring hyperbaric oxygen therapy, which was recorded as a late G3 GU toxicity. One patient experienced bleeding from the biopsied rectum site, which was recorded as a late G2 GI toxicity. GU and GI toxicities of grade 4 or greater were not observed.

\section{Discussion}

The present study aimed to evaluate clinical outcomes of CIRT for prostate cancer at our institute with special regard to toxicities and determine the efficacy of our treatment protocol for localized prostate cancer. In particle therapy, beam characteristics and parameters differ by institutions because treatment planning systems and beam modification devices differ by institutions; therefore, the validation of treatment results is highly important to establish CIRT as a safe and effective treatment method. The CIRT dose fractionation was determined as 57.6 Gy (RBE) in 16 fractions over 4 weeks. This dose fractionation was recommended through prostate cancer treatment in NIRS [7]. The methods of patient fixation and basic design of PTV were similar to that used in NIRS.

Excellent treatment results with a low incidence of adverse events have been previously reported [7]. 1Ishikawa et al. reviewed the clinical outcomes of CIRT for prostate cancer over the last decade at NIRS, where CIRT was initiated for prostate cancer in 1995, and several phase I/II studies were performed to establish radiotherapy technique and determine the optimal radiation dose. Hypofractionation is also carried out; a total dose of $57.6 \mathrm{~Gy}$ $(\mathrm{RBE})$ in 16 fractions was given over 4 weeks is almost equivalent based on the L/Q model (assuming $\alpha / \beta$ of prostate cancer cells is 2) to $63 \mathrm{~Gy}(\mathrm{RBE})$ in 20 fractions over 5 weeks. Furthermore, $51.6 \mathrm{~Gy}$ (RBE) in 12 fractions over 3 weeks is currently used [15]. They analyzed 927 patients, who have been followed up for at least 6 months. The 5-year biochemical relapse-free and LCRs were 90.6 and $98.3 \%$, respectively. The 5 -year biochemical relapsefree rates of the low-, intermediate-, and high-risk groups were $89.6,96.8$, and $88.4 \%$, respectively.

Although excellent clinical results of CIRT for prostate cancer were demonstrated, they were reported only from one facility. Therefore, a multi-institutional retrospective study was conducted [16] to overcome this limitation. Data of 2157 patients enrolled in the prospective studies of 3 CIRT institutions were analyzed. The 5-year biochemical recurrence-free survival in low-, intermediate-, and high-risk patients was 92, 89, and 92\%, respectively. Grade 3 or more toxicity was not observed. Thus, excellent treatment results with low toxicity were confirmed. However, this multi-institutional study was a retrospective study, and the observation period was only 29 months.

Our present analysis is the first prospective observational study conducted at a facility other than NIRS, with an observation period of 60 months. The 5-year bRFRs were 91.7, 93.4, and 92.0\% in low-, intermediate-, and high-risk patients, respectively. Late grades 2 and 3 genitourinary and gastrointestinal toxicity rates were 9.0 and $0.3 \%$ and 0.3 , and $0 \%$, respectively. Therefore, this prospective study was the first to be conducted outside NIRS and to reproduce the previously reported treatment results, with a reasonably long follow up.

Recently, several clinical results of hypofractionated Xray IMRT trials were reported. Randomized studies which compare hypofractionated schedule $60 \mathrm{~Gy}$ in 20 fractions (equivalent dose in $2 \mathrm{~Gy}$ fractions assuming $\alpha / \beta=2$ (EQD2) 75 Gy) with conventional fractionations (78 Gy in 39 fractions or 74 Gy in 37 fractions) showed that 5 -year biochemical failure free rates of hypofractionated arms were $85-90.6 \%$ meeting noninferiority criteria without 
$\mathbf{a}$

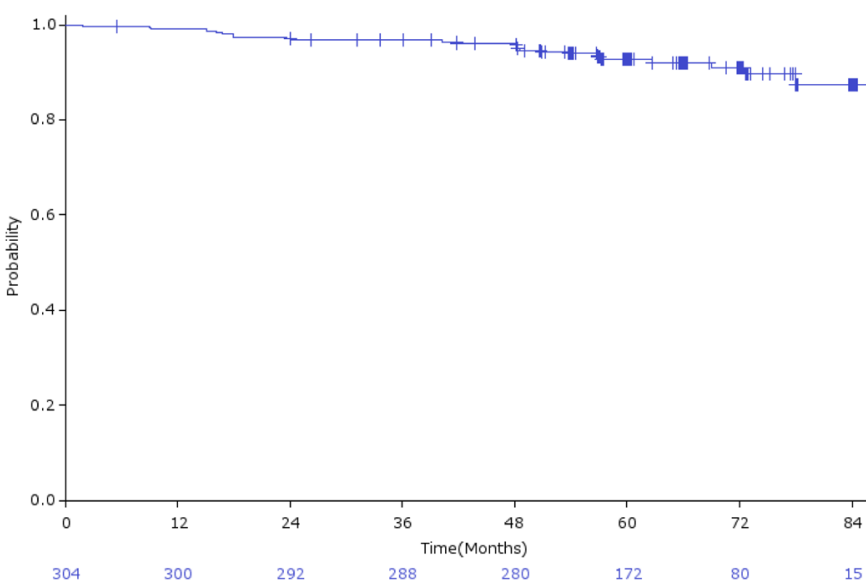

b
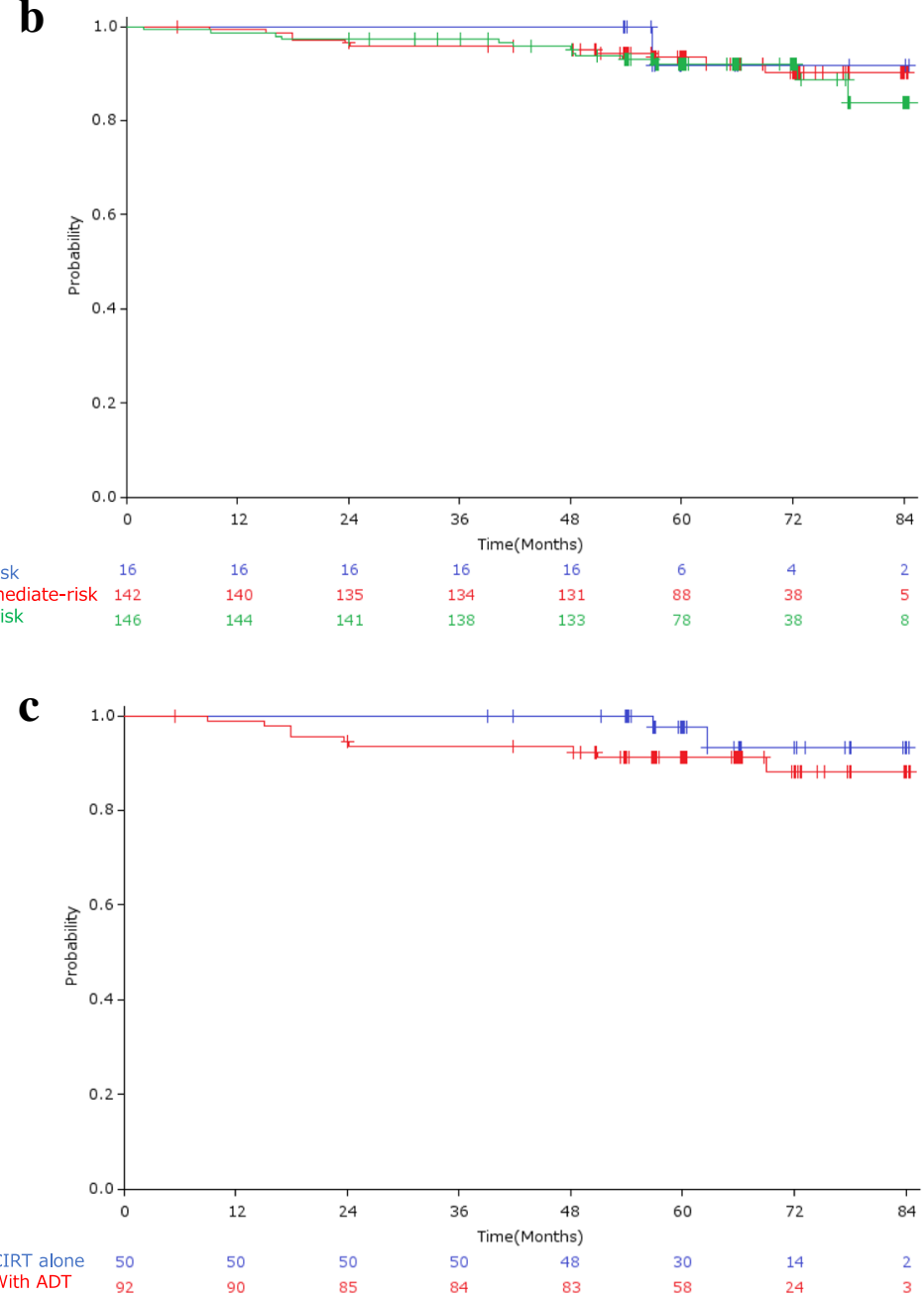

Fig. 2 a) Biochemical relapse-free rate; b) Biochemical relapse-free rate by risk group; c) Biochemical relapse-free rate by use of ADT in intermediate-risk group patients

significant increase of late toxicity $[17,18]$. Another study compared slightly higher dose $64.6 \mathrm{~Gy}$ in 19 fractions (EQD2 87.2 Gy) with conventional 78 Gy in 39 fractions and 5-year biochemical failure free rate was $80 \%$ and was not superior than conventional arm with slightly higher GI toxicity $[19,20]$. Of course, it is difficult to compare 


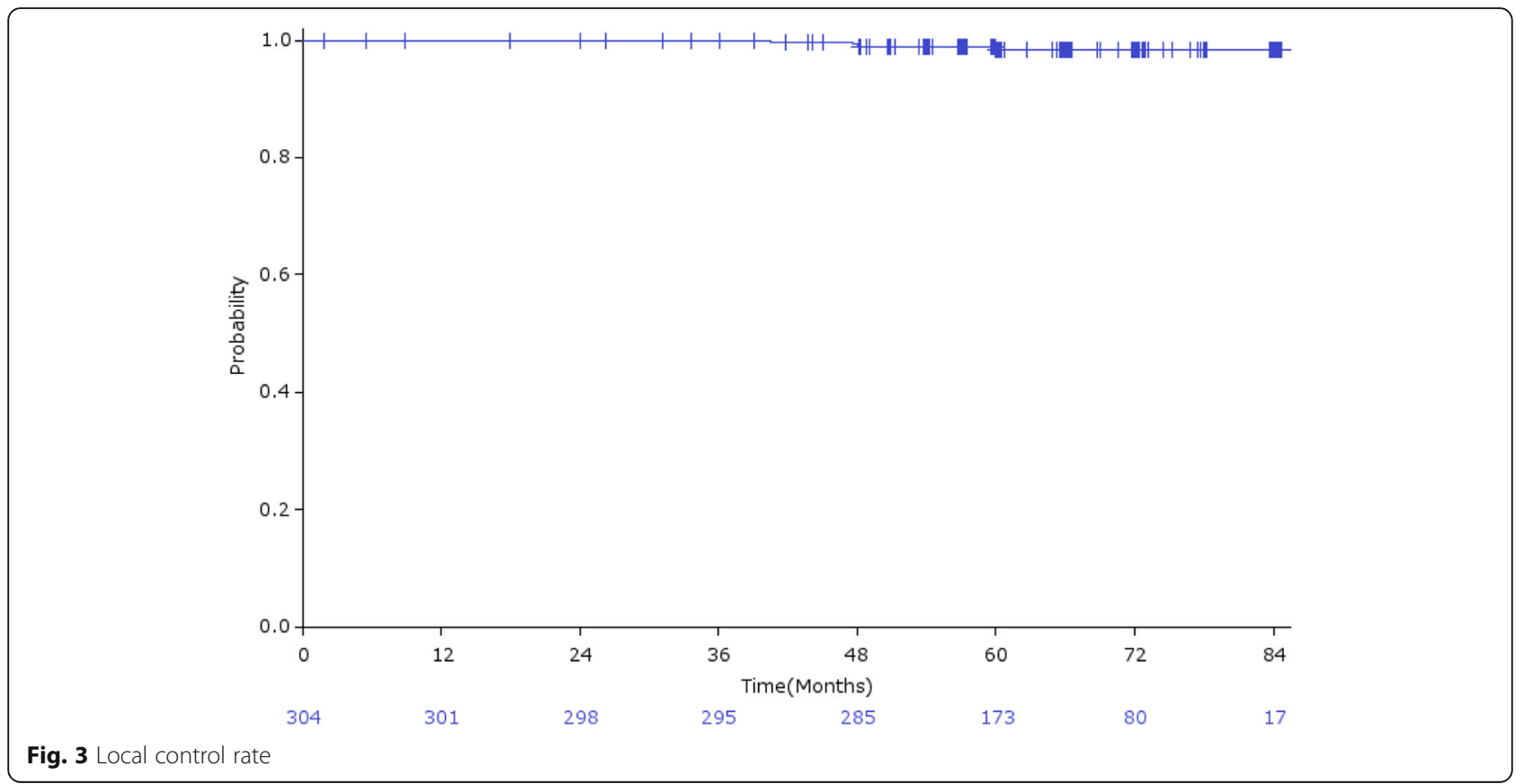

these results with this current study because of differences in patients' risk group, protocol design, $\mathrm{ADT}$ regimen and so on, our fractionation schedule 57.6 Gy (RBE) in 16 fractions (EQD2 81 Gy) CIRT shows reasonable 5-year biochemical relapse-free rate of $90.6 \%$ and promising GI toxicity rate with short treatment period.

Furthermore, diagnosis and treatment in the present study were homogenous, with the GS in all patients confirmed by a central pathological review. A recent study reported wide discordance in biopsy Gleason grading in one- third of all cases [21]. In the present study, CIRT was performed as planned in all patients, and hormonal therapy was planned in advance and performed with few protocol deviations. Of the 304 analyzed patients, only three patients suspended ADT. Therefore, the present study protocol demonstrated acceptable tolerability and quality.

In addition, we performed CIRT without ADT in favorable intermediate-risk patients. The definition of "favorable" intermediate-risk patients was not established at the time of this protocol design; therefore, this definition

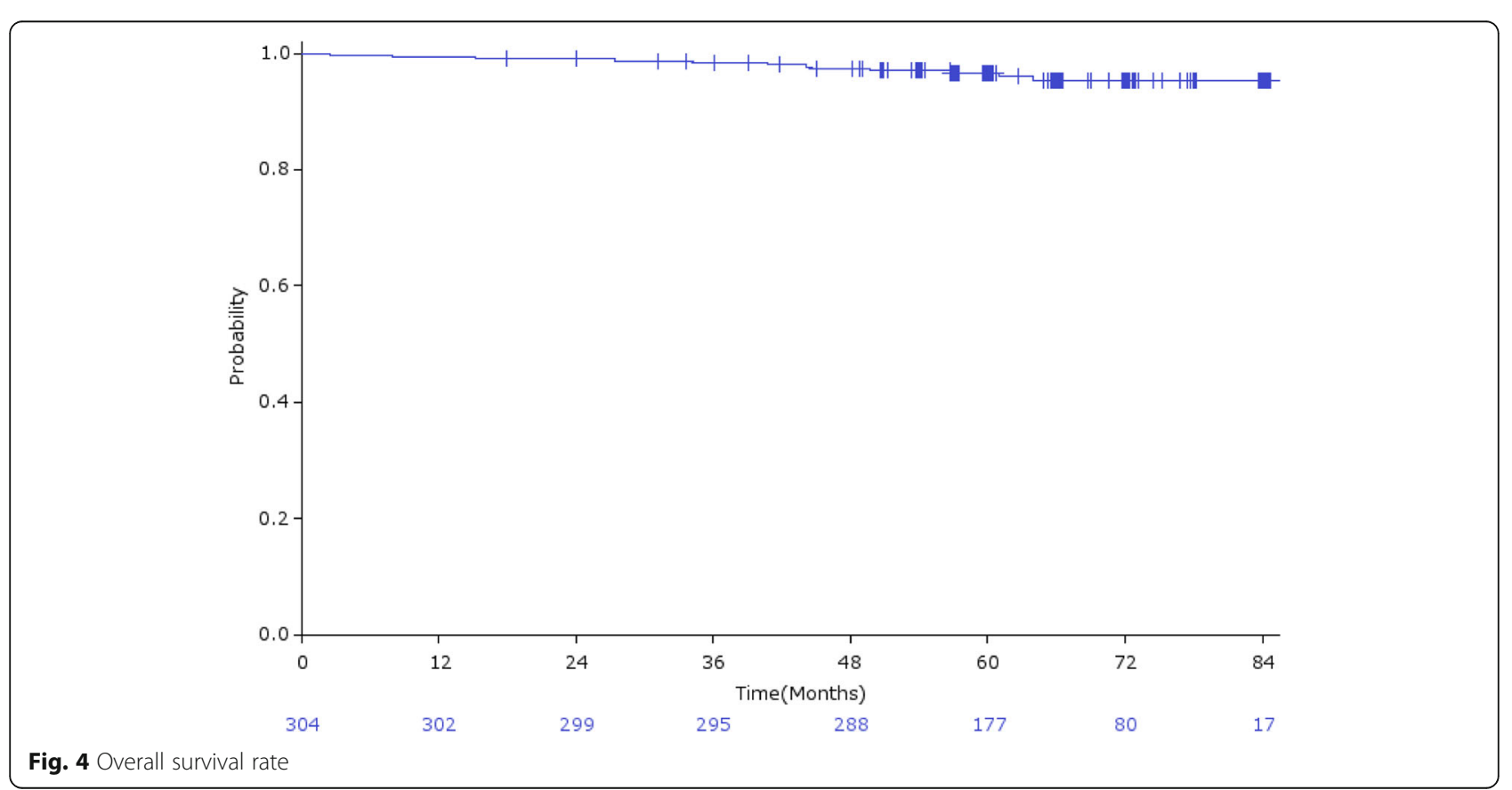


Table 2 Acute and late toxicity

\begin{tabular}{|c|c|c|c|c|c|c|c|c|}
\hline & \multicolumn{4}{|c|}{ Genitourinary toxicity } & \multicolumn{4}{|c|}{ Gastrointestinal toxicity } \\
\hline & G0 & G1 & G2 & $\geqq G 3$ & G0 & G1 & G2 & $\geqq G 3$ \\
\hline Acute & $131(43 \%)$ & $160(53 \%)$ & $13(4 \%)$ & $0(0 \%)$ & 300 (99\%) & $4(1 \%)$ & $0(0 \%)$ & $0(0 \%)$ \\
\hline Late Maximum & 118 (39\%) & $156(52 \%)$ & $28(9 \%)$ & $1(0.3 \%)$ & 276 (91\%) & $26(9 \%)$ & $1(0.3 \%)$ & $0(0 \%)$ \\
\hline Late Last Follow up & 236 (78\%) & $58(19 \%)$ & $9(3 \%)$ & $0(0 \%)$ & 302 (99.7\%) & 1 (0.3\%) & $0(0 \%)$ & 0 (0\%) \\
\hline
\end{tabular}

differs slightly from recent guidelines (e.g., the NCCN guideline). With our definition, favorable intermediaterisk patients demonstrated good PSA control without ADT. This result validates the efficacy of CIRT in "favorable" intermediate-risk patients with our definition.

The limitation of this study is that the median follow-up period of 60 months was relatively short in considering the long natural history of prostate cancer. Therefore, additional follow-up studies are required to evaluate the final outcomes of the present study.

\section{Conclusions}

In conclusion, the present prospective study demonstrates excellent PSA control with a low incidence of severe toxicity following CIRT for prostate cancer. These outcomes are similar to those previously reported for CIRT, indicating reproducible efficacy. The present study may contribute to the establishment of CIRT as a one of the effective modality for the treatment of prostate cancer.

\section{Abbreviations}

ADT: Androgen deprivation therapy; CIRT: Carbon ion radiotherapy; GHMC: Gunma University Heavy lon Medical Center; Gl: Gastrointestinal; GU: Genitourinary; NIRS: National Institute of Radiological Sciences;

PSA: prostate-specific antigen

\author{
Acknowledgements \\ Group for Genitourinary Tumors at Gunma University Heavy Ion Medical \\ Center. \\ Tel: + 81-27-220-8383; Fax: + 81-27-220-8397. \\ 3-39-22, Showa-machi, Maebashi, Gunma, 371-8511 Japan. \\ Principal investigator: \\ Kazuhiro Suzuki: Gunma University, Gunma, Japan. \\ Co-investigators: \\ Kazuto Ito: Gunma University, Gunma, Japan. \\ Nobuaki Shimizu: Gunma Cancer Center, Gunma, Japan. \\ Yutaka Takezawa: Isesaki Municipal Hospital, Gunma, Japan. \\ Junko Hirato: Gunma University, Gunma, Japan. \\ Hitoshi Ishikawa: Tsukuba University, Ibaraki, Japan. \\ Hiroshi Matsui: Gunma University, Gunma, Japan. \\ Hiroyuki Katoh: Kanagawa Cancer Center, Japan. \\ Tatsuya Ohno: Gunma University, Gunma, Japan. \\ Hidemasa Kawamura: Gunma University, Gunma, Japan.
}

\section{Authors' contributions}

Conceptualization: KS, TO, TN. Data curation and analysis: HK, NK, HS, TM, HK, HI, HM. Supervision: KI, KS, HK, HI, TO, TN. Writing - original draft: HK. Review and editing: HK, KI, KS, TO, TN. All authors have read and approved the manuscript.

\section{Funding}

No funding was obtained for this study.

\section{Availability of data and materials}

The datasets generated and analyzed during the current study are not publicly available due to including personal information but are available from the corresponding author on reasonable request.

\section{Ethics approval and consent to participate}

The present study protocol was designed by the Working Group of Genitourinary Tumors of GHMC. The protocol was approved by the institutional ethical committee" Gunma university hospital institutional review board", approval number 693, and was registered with the University Medical Information Network Clinical Trial Registry, number UMIN000003827. All patients signed informed consent forms.

\section{Consent for publication}

Not Applicable.

\section{Competing interests}

The authors declare that they have no competing interests.

\section{Author details}

${ }^{1}$ Gunma University Heavy Ion Medical Center, 3-39-22, Showa-machi, Maebashi, Gunma 371-8511, Japan. ²Department of Radiation Oncology, Gunma University Graduate School of Medicine, Maebashi, Gunma, Japan. ${ }^{3}$ Ion-beam Radiation Oncology Center, Kanagawa Cancer Center, Yokohama, Kanagawa, Japan. ${ }^{4}$ Department of Radiation Oncology, Faculty of Medicine, University of Tsukuba, Tsukuba, Ibaraki, Japan. ${ }^{5}$ Department of Urology, Gunma University Graduate School of Medicine, Maebashi, Gunma, Japan. ${ }^{6}$ Institute for Preventive Medicine, Kurosawa Hospital, Maebashi, Gunma, Japan.

Received: 29 March 2019 Accepted: 23 January 2020

Published online: 30 January 2020

\section{References}

1. International Agency for Research on Cancer IARC Prostate Cancer. Estimated Incidence, Mortality and Prevalence Worldwide in 2012. http:// globocan.iarc.fr/Pages/fact_sheets_cancer.aspx.

2. Ito K. Prostate cancer in Asian men. Nat Rev Urol. 2014;11:197-212.

3. Onozawa M, Hinotsu S, Tsukamoto T, Oya M, Ogawa O, Kitamura T, et al. Recent trends in the initial therapy for newly diagnosed prostate cancer in Japan. Jpn J Clin Oncol. 2014;44:969-81.

4. Georg D, Hopfgartner J, Gòra J, Kuess P, Kragl G, Berger D, et al. Dosimetric considerations to determine the optimal technique for localized prostate cancer among external photon, proton, or carbon-ion therapy and high-dose-rate or low-dose-rate brachytherapy. Int J Radiat Oncol Biol Phys. 2014;88:715-22.

5. Held KD, Kawamura H, Kaminuma T, Paz AS, Yoshida Y, Liu Q, et al. Effects of Charged Particles on Human Tumor Cells. Front Oncol. 2016;6(Suppl 1):23.

6. Durante M, Orecchia R, Loeffler JS. Charged-particle therapy in cancer: clinical uses and future perspectives. Nat Rev Clin Oncol. 2017;14:483-95.

7. Ishikawa H, Tsuji H, Kamada T, Akakura K, Suzuki H, Shimazaki J, et al. Carbonion radiation therapy for prostate cancer. Int J Urol. 2012;19:296-305.

8. Ohno T, Kanai T, Yamada S, Yusa K, Tashiro M, Shimada H, et al. Carbon Ion Radiotherapy at the Gunma University Heavy Ion Medical Center: New Facility Set-up. Cancers. 2011;3:4046.

9. Okada T, Okada T, PhD H, Tsuji H, PhD T, Kamada T, et al. Carbon ion radiotherapy in advanced hypofractionated regimens for prostate cancer: from 20 to 16 fractions. Int J Radiat Oncol Biol Phys. 2012;84:968-72.

10. Nakamura K, Mizowaki T, Imada H, Karasawa K, Uno T, Onishi H, et al. External-beam radiotherapy for localized or locally advanced prostate cancer in Japan: a multi-institutional outcome analysis. Jpn J Clin Oncol. 2008;38:200-4. 
11. Akakura K, Tsujii H, Morita S, Tsuji H, Yagishita T, Isaka S, et al. Phase I/Il clinical trials of carbon ion therapy for prostate cancer. Prostate. 2004;58:252.

12. Tsuji H, Yanagi T, Ishikawa H, Kamada T, Mizoe J, Kanai T, et al. Hypofractionated radiotherapy with carbon ion beams for prostate cancer. Int J Radiat Oncol Biol Phys. 2005;63:1153-60.

13. Ishikawa H, Tsuji H, Kamada T, Yanagi T, Mizoe J-E, Kanai T, et al. Carbon ion radiation therapy for prostate cancer: results of a prospective phase II study. Radiother Oncol. 2006;81:57-64.

14. Roach M III, Hanks G, Thames H Jr, Schellhammer P, Shipley WU, Sokol GH et al. Defining biochemical failure following radiotherapy with or without hormonal therapy in men with clinically localized prostate cancer: Recommendations of the RTOG-ASTRO Phoenix Consensus Conference. Int J Radiat Oncol Biol Phys. 2006;65:965-74.

15. Nomiya T, Tsuji H, Maruyama K, Toyama S, Suzuki H, Akakura K, et al. Phase I/II trial of definitive carbon ion radiotherapy for prostate cancer: evaluation of shortening of treatment period to 3 weeks. Brit J Cancer. 2014;1 10:2389-95.

16. Nomiya T, Tsuji H, Kawamura H, Ohno T, Toyama S, Shioyama Y, et al. A multi-institutional analysis of prospective studies of carbon ion radiotherapy for prostate cancer: A report from the Japan Carbon ion Radiation Oncology Study Group (J-CROS). Radiother Oncol. 2016;121:288-93.

17. Catton CN, Lukka H, Gu C-S, Martin JM, Supiot S, Chung PW, et al. Randomized Trial of a Hypofractionated Radiation Regimen for the Treatment of Localized Prostate Cancer. J Clin Oncol. 2017;35:1884-90.

18. Dearnaley D, Syndikus I, Mossop H, Khoo V, Birtle A, Bloomfield D, et al. Conventional versus hypofractionated high-dose intensity-modulated radiotherapy for prostate cancer: 5-year outcomes of the randomised, noninferiority, phase 3 CHHiP trial. Lancet Oncol. 2016;17:1047-60.

19. Incrocci L, Wortel RC, Alemayehu W, Aluwini S, Schimmel E, Krol S, et al. Hypofractionated versus conventionally fractionated radiotherapy for patients with localised prostate cancer (HYPRO): final efficacy results from a randomised, multicentre, open-label, phase 3 trial. Lancet Oncol. 2016;17:1061-9.

20. Aluwini S, Pos F, Schimmel E, Krol S, van der Toorn P, de Jager H, et al. Hypofractionated versus conventionally fractionated radiotherapy for patients with prostate cancer (HYPRO): late toxicity results from a randomised, non-inferiority, phase 3 trial. Lancet Oncol. 2016:17:464-74.

21. Sasaki H, Kido M, Miki K, Aoki M, Takahashi H, Dokiya T, et al. Results of central pathology review of prostatic biopsies in a contemporary series from a phase III, multicenter, randomized controlled trial (SHIP0804). Pathol Int. 2015;65:177-82.

\section{Publisher's Note}

Springer Nature remains neutral with regard to jurisdictional claims in published maps and institutional affiliations.

Ready to submit your research? Choose BMC and benefit from:

- fast, convenient online submission

- thorough peer review by experienced researchers in your field

- rapid publication on acceptance

- support for research data, including large and complex data types

- gold Open Access which fosters wider collaboration and increased citations

- maximum visibility for your research: over $100 \mathrm{M}$ website views per year

At $\mathrm{BMC}$, research is always in progress.

Learn more biomedcentral.com/submissions 